\title{
Reflets
}

Revue d'intervention sociale et communautaire

\section{Une réalité qu'on ne peut ignorer : portrait régional des violences faites aux femmes des comtés de Prescott-Russell et les services communautaires disponibles pour les femmes et les familles. Récit d'une recherche-action}

\section{Cécile Coderre}

Volume 22, numéro 2, automne 2016

URI : https://id.erudit.org/iderudit/1038975ar

DOI : https://doi.org/10.7202/1038975ar

Aller au sommaire du numéro

Éditeur(s)

Reflets, Revue d'intervention sociale et communautaire

ISSN

1203-4576 (imprimé)

1712-8498 (numérique)

Découvrir la revue

Citer cet article

Coderre, C. (2016). Une réalité qu'on ne peut ignorer : portrait régional des violences faites aux femmes des comtés de Prescott-Russell et les services communautaires disponibles pour les femmes et les familles. Récit d'une recherche-action. Reflets, 22(2), 155-173. https://doi.org/10.7202/1038975ar 


\title{
Une réalité qu'on ne peut ignorer : portrait régional des violences faites aux femmes des comtés de Prescott-Russell et les services communautaires disponibles pour les femmes et les familles. Récit d'une recherche-action
}

\author{
Cécile Coderre, Ph.D. \\ École de service social, Université d'Ottawa
}

Dans la foulée de la création de ressources pour les femmes vivant des expériences de violence, outre les services directs tels que les maisons d'hébergement, les centres de lutte contre les violences sexuelles et les programmes pour les enfants exposés à la violence conjugale, de nombreuses coalitions regroupant des organismes communautaires sur une base régionale ont vu le jour en Ontario afin de favoriser les partenariats dans la lutte contre la violence faite aux femmes. Afin de mieux comprendre les enjeux régionaux et de réfléchir à l'adéquation entre les services offerts et les besoins des femmes, deux de ces coalitions régionales, soit la Coalition d'Ottawa contre la violence faite aux femmes et The Niagara Coalition to End Violence Against Women, ont préparé en 2008 des portraits régionaux et développé des stratégies pour améliorer les services offerts aux femmes. C'est à ce projet que s'est attaquée, en 2012, la Coalition de Prescott-Russell pour éliminer la violence faite aux femmes. Nous présenterons brièvement la recherche-action que nous avons menée dans le cadre de cette initiative, de même que les principaux résultats de cette recherche.

\section{Une recherche communautaire}

La Coalition de Prescott-Russell pour éliminer la violence faite aux femmes est un organisme communautaire très dynamique de la région de Prescott-Russell. Créée en 2007 et regroupant 14 organismes, la Coalition a obtenu une subvention du ministère des Services sociaux et communautaires de l'Ontario « dans le but de sensibiliser et éduquer le 
public au sujet de la violence faite aux femmes (VFF) et de promouvoir la collaboration et la coordination des organismes de la collectivité " (Coalition, 2014, p. 8). L'analyse féministe de la violence faite aux femmes est inscrite dans ses valeurs tout comme l'égalité, l'inclusion et la diversité.

En 2011, la Coalition inscrit dans ses projets de développement la création d'un outil régional de collecte de données sur les violences faites aux femmes afin d'avoir un portrait régional plus juste et de lui permettre d'exercer pleinement un de ses mandats à savoir : "renforcer les partenariats et l'entraide entre organismes en partageant de l'information, des ressources et de l'expertise " (Coalition, 2014, p. 8). C'est ainsi qu'en décembre 2011, une première présentation d'un projet de recherche participative est soumise aux membres de la Coalition lors de la rencontre trimestrielle. D'emblée ce projet suscite l'intérêt des représentantes de tous les organismes et, en mai 2012, un comité encadreur composé de 6 membres est créé. Il commence à se réunir à l'automne de la même année. Ce comité comprenait : Anne Jutras, directrice générale du Centre Novas - CALACS francophone de Prescott-Russell; Rachel Lamoureux, coordonnatrice de la Coalition de Prescott-Russell pour éliminer la violence faite aux femmes; Céline Pelletier, directrice générale de Maison Interlude; Renée Proulx-Lamarche, psychothérapeute et chef en Violence et Agression sexuelle, Centre Royal-Comtois - Hôpital Général de Hawkesbury; Chantale Tremblay, directrice du Centre de services de Hawkesbury, Valoris pour enfants et adultes de Prescott-Russell; Cécile Coderre, professeure, École de service social, Université d'Ottawa. De plus, Julie Delorme, une étudiante à la maîtrise de l'École de service social, a participé aux travaux du comité encadreur, tant à la collecte et à l'analyse des données qu'à la rédaction du rapport.

L'expérience de la Coalition d'Ottawa contre la violence faite aux femmes, qui a publié deux éditions d'une étude sur la prévalence de la violence faite aux femmes d'Ottawa, soit À l'abri des regards, volume I (2009) et volume II (2011), a été précieuse dans le développement de certains outils de collecte de données. Nous avons ainsi adapté un de ses instruments de collecte, le sondage éclair, à la couleur régionale des comtés. Nous avons aussi créé d'autres outils, dont quatre questionnaires pour réaliser des groupes d'entrevues auprès d'intervenantes et intervenants : le premier touchant les dynamiques des violences conjugale et sexuelle vécues en milieu rural; le deuxième ciblant spécifiquement les jeunes filles; le troisième portant sur les principales raisons de consulter les services, les problèmes les plus fréquents et les solutions à envisager concernant surtout les services d'intervention judiciaire et la garde des enfants; le quatrième ayant trait aux défis que doivent relever les femmes. Nous avons aussi mis en place un mécanisme de collecte de 
données sur la population utilisatrice de tous les services offerts pour contrer les violences faites aux femmes pour les années 2011-2012 et 2012-2013. Il sera donc possible dans les années futures de comparer l'offre de services.

Après l'élaboration d'un calendrier de travail pour chacune des étapes de la collecte des données, calendrier approuvé par les membres de la Coalition, tous les documents, que ce soit les questionnaires, le sondage éclair, les lettres pour les demandes de participation, les formulaires de consentement et de collecte des données, l'analyse des résultats et les sections touchant les services offerts, ont été vérifiés et validés par le comité encadreur. Ce comité a également effectué un travail de relecture des différentes versions du rapport et approfondi la réflexion sur le titre du rapport : Une réalité qu'on ne peut ignorer : Portrait régional des violences faites aux femmes des comtés de Prescott-Russell et les services communautaires disponibles pour les femmes et les familles. Par ailleurs, durant les deux années du projet, un suivi de la recherche a été présenté aux membres de la Coalition lors des rencontres trimestrielles, et les recommandations ont été approuvées lors d'une réunion de la Coalition. C'est le sondage éclair qui a mobilisé le plus de ressources puisque plus de 30 intervenantes ont été investies du titre d'enquêtrice auprès de leur organisme respectif pour une période de 3 à 5 jours. Comme tout le travail de collecte et de rédaction a été fait sur une base bénévole tant par les membres du comité encadreur que par les intervenantes dans tous les organismes participants, il était nécessaire de bien connaitre les enjeux de l'organisation du travail de chaque organisme participant. Il faut reconnaître que les directions de tous les organismes ont été partie prenante de cet exercice et, ainsi, se sont engagées directement dans un réel partenariat entre organismes en vue de "déterminer les lacunes [...] pour améliorer l'accès aux services et répondre aux besoins de la communauté " (Coalition, 2014, p. 8).

Lors de la préparation du plan du rapport final, il semblait important de faire connaitre les résultats de la recherche sur le terrain, mais il apparaissait tout aussi nécessaire de documenter plus généralement les connaissances sur les différentes formes de violence faite aux femmes, y compris dans la reconnaissance de la diversité des expériences. Si les dynamiques de la violence en milieu rural ont pris plus d'ampleur, c'est aussi parce qu'il s'agit du terreau quotidien des femmes des comtés et que ces dynamiques sont moins connues et documentées. Cependant, les milieux ruraux sont aussi touchés par la diversité des expériences des femmes selon qu'elles sont jeunes, plus âgées, atteintes d'un handicap ou tout simplement touchées par un problème de santé mentale, ou qu'elles sont des assistées sociales ou des personnes à faible revenu, des Autochtones, des francophones ou des anglophones, ou encore des immigrantes nouvellement arrivées. Bien qu'il n'ait 
pas été possible de faire un portrait détaillé de chacune de ces différentes réalités dans les comtés, leur prise en considération lors de la mise en place des services est devenue essentielle. C'est pourquoi nous avons fait une synthèse des connaissances actuelles sur ces différentes problématiques en plus de souligner les contributions des organismes du milieu. C'est aussi une manière de répondre à un des mandats de la Coalition, soit celui de "s'éduquer sur la problématique de la violence faite aux femmes, sur les meilleures pratiques et recherches récentes" (Coalition, 2014, p. 8). Nous avons aussi présenté de manière exhaustive les services offerts aux femmes et aux familles touchées par la violence, les programmes spécifiques des organismes ainsi que les activités régulières ou ponctuelles mises en place par ceux-ci. Et ce, sans oublier les activités de prévention de la violence faite aux femmes, telles que les marches à talons hauts ou encore les 10 jours pour l'élimination de la violence. En regroupant dans un seul document (accessible en ligne ou encore au sein des organismes membres) toutes les ressources disponibles, il est possible aussi de répondre à un autre des mandats de la Coalition, à savoir « renforcer les partenariats et l'entraide [...] en partageant de l'information, des ressources et de l'expertise "(Coalition, 2014, p. 8).

\section{Les principaux résultats de la recherche sur les violences faites aux femmes dans les comtés de Prescott-Russell}

Partageons maintenant les grands résultats de cette recherche. En premier lieu, nous dévoilons ce que nous avons appris au sujet de la dynamique de la violence faite aux femmes en milieu rural dans les comtés de Prescott-Russell. En deuxième lieu, nous présentons ce que nous retenons de l'expérience des femmes qui utilisent les services dans la région. En troisième lieu, nous soulevons quelques éléments qui ont découlé de la recherche en matière de collaboration et de connaissances sur les besoins des femmes vivant des situations de violence en milieu rural.

\section{Les dynamiques des violences en milieu rural}

En premier lieu, analysons les dynamiques des violences faites aux femmes en milieu rural. Il est difficile de s'imaginer la vie en région éloignée ou en milieu rural à partir de l'expérience urbaine. La population rurale dépend grandement de la voiture afin d'accéder à divers services de santé, d'employabilité ou d'éducation. De plus, on ne peut compter sur 
la présence de services de la même façon qu'en milieu urbain. Les services policiers sont souvent assurés par un détachement provincial dont le point de service peut être éloigné de la communauté. Même situation pour les établissements de santé qui, bien souvent, ne peuvent offrir des services de façon quotidienne. C'est donc dire que les femmes victimes de violence en milieu rural font face à des obstacles de taille en ce qui a trait à l'accès aux services. Pour ce qui est de la violence envers les femmes, cet état de fait accentue leur vulnérabilité. Les femmes en milieu rural font l'expérience d'un isolement lié non seulement à la violence, mais aussi à l'éloignement. Et cet isolement est aggravé par le caractère homogène des milieux ruraux. Cette homogénéité est consolidée par les valeurs patriarcales, les questions religieuses, le déni de la communauté face à la violence faite aux femmes, les pressions liées à la conformité sociale et l'absence d'anonymat (Dragiewicz et DeKeseredy, 2008; DeKeseredy, Dragiewicz et Rennison, 2012; DeKeseredy et Schwartz, 2009; Doherty, 2002; Doherty et Hornosty, 2008; Jiwani, Kachuk et Moore, 1998; Kasdorff et Erb, 2010; Martz et Bryson Sarauer, 2001; Peek-Asa, et collab., 2011; Riddell, Ford-Gilboe et Leipert, 2008).

Pour comprendre les problèmes que les femmes en milieu rural rencontrent lorsqu' elles vivent des situations de violence, il est très important de retenir deux concepts : l'urbanicentrisme et le patriarcat rural. L'urbanicentrisme se caractérise par l'assimilation du vécu des femmes rurales à celui des femmes urbaines, c'est-à-dire en interprétant la réalité des femmes victimes de violence en milieu rural selon le contexte de la violence que subissent les femmes en milieu urbain. Bien entendu, comme le soulignent Hornosty et Doherty (2001, p. 9), les formes que peut prendre la violence peuvent être similaires, mais c'est le contexte communautaire qui déterminera les différences entre rural et urbain. À cet égard, Brownridge (2009) et DeKeseredy et Schwartz (2009) soulignent qu'en milieu rural les normes communautaires pour interdire aux femmes de révéler leur expérience de violence et ainsi de chercher un support social sont très prégnantes. D'autre part, l'urbanicentrisme se résume aussi à l'idéalisation de la vie en campagne, vision qui tend à cacher tout problème social. Ce préjugé favorable tend à imposer une vision de la réalité rurale comme un bloc monolithique, ignorant la diversité présente en milieu rural.

Ainsi, il n'existe pas un milieu rural, mais des milieux ruraux. Cela ne veut pas dire cependant qu'à l'intérieur de ces communautés, on ne retrouve pas une plus grande homogénéité qu'en ville. Une composante importante qui forme le caractère homogène des collectivités rurales est liée, selon Hornosty et Doherty (2002, p. 8), au concept de " patriarcat rural ». Ce concept est défini par Websdale comme suit : 
Un groupe de valeurs, croyances et idées collectives qui sous-tendent que les femmes en milieu rural sont inférieures ou sont les subordonnées des hommes en milieu rural. [notre traduction] (Websdale, 1998, p. 48, cité dans Jiwani, 1998)

Ce n'est pas que les croyances patriarcales soient particulières au milieu rural ou qu'elles soient absentes en milieu urbain. Ces croyances en milieu urbain cohabitent avec d'autres visions, et cette cohabitation crée une plus grande diversité de visions. De ce fait, les croyances en l'infériorité des femmes en milieu rural, soutenues par l'éloignement géographique et par le comportement contrôleur de leur partenaire abusif, contribuent ainsi à l'invisibilité de la violence en milieu rural. Associée au patriarcat rural, l'utilisation des armes à feu est aussi plus fréquente et pourrait constituer, selon des chercheurs, un mécanisme de contrôle, un outil de menace utilisé contre les femmes (DeKeseredy et Schwartz, 2009; Dragiewicz et DeKeseredy, 2008).

Les intervenantes rencontrées ont confirmé que la violence conjugale est encore un tabou dans les comtés. À ce titre, elles soulignent que c'est une histoire qui se règle en famille et que, même pour la dévoiler, l'isolement peut faire en sorte que personne ne peut l'entendre.

«Les gens sont plus privés, dans les milieux ruraux, les gens gardent des choses beaucoup plus privées [...] Ils ont moins d'opportunité de partager ça avec une voisine, à l'épicerie, ou quelqu'un au dépanneur, donc tout reste privé; ça les garde [...] dans le secret. »

«L'isolement par rapport au fait qu'il n'y a pas de voisins si proches. Quand tu vis sur une ferme, comme ce sont des entreprises avec plus d'une génération, leur réseau, c'est leur famille. Elles sont isolées de tout et manquent de contact avec les autres réseaux de support. "

Ainsi, la situation se complexifie lorsque le seul réseau de soutien est le réseau familial. Le manque de transport, problème endémique dans les comtés, augmente les risques d'isolement et réduit la possibilité d'avoir accès à des services, et même d'y accéder de façon confidentielle. Par exemple, comment se faire reconduire à un service d'aide par le conjoint agresseur ou un membre de sa famille si l'on veut assurer sa sécurité? Afin de prendre la mesure de la problématique de l'accès aux services en violence dans les comtés, nous avons conçu une carte mettant en relief les distances entre les services et les principales municipalités. Il ressort de cet exercice que, pour avoir accès au programme 
d'aide aux victimes et aux témoins à L'Orignal, les résidentes d'Embrun doivent parcourir 158 kilomètres (aller-retour), ou encore pour accéder aux services du Centre Novas à Casselman, les femmes qui résident à Rockland doivent parcourir de 70 à 90 kilomètres. En raison de l'absence de transport en commun et des coûts de taxi prohibitifs pour de si longues distances, le recours aux services est en quelque sorte dénié. Et que dire de la confidentialité dans la situation où il faut attendre dans la même salle d'attente que son voisin dans un quelconque service.

«C'est que pour aller cogner à la porte d'un service, c'est difficile, surtout si tu ne veux pas rencontrer ton voisin."

"Souvent les gens viennent me voir à Casselman ou à Rockland, pour ne pas venir à Hawkesbury, pour ne pas être vus dans la salle d'attente à Hawkesbury. "

Certaines caractéristiques particulières du milieu rural et semi-rural ont aussi un impact significatif lorsque la femme doit quitter son domicile pour assurer sa sécurité. Globalement, les résidents des comtés unis sont très majoritairement propriétaires de leur logement (83\%). Le revenu annuel des femmes en 2006 était inférieur de 13637 \$ à celui des hommes, et leur taux d'emploi est inférieur à celui des hommes, bien qu'elles détiennent davantage que les hommes un diplôme postsecondaire. Ainsi, les femmes confrontées à des expériences de violence conjugale doivent souvent faire des " choix " déchirants ou carrément impossibles pour assurer leur sécurité, notamment si elles doivent quitter le foyer avec leurs enfants. Comment vivre en sécurité sans devoir abandonner sa sécurité financière et souvent l'entreprise familiale? Comment refaire sa vie sans violence, mais sans autonomie financière? Comment se trouver un logement locatif dans une région où c'est le règne des résidences privées? Puisque le marché locatif est concentré à Hawkesbury, cela implique souvent, pour la majorité des résidentes et leurs enfants, la nécessité d'abandonner aussi leur milieu de vie, l'école, les amies et amis et les activités sportives pour les enfants.

"La même chose si tu as un emploi, le logement, c'est pas évident; tu pars d'un village à un autre. Il y a peu de logements locatifs. "

"Au niveau de l'entreprise familiale, trois quarts du temps, c'est elle qui fait rouler la partie administrative de l'entreprise. Elle doit se trouver un autre emploi, quelquefois sans qualification précise. " 
La question du logement abordable est critique dans les comtés. Un comité régional s'est penché sur cette question en 2013 et, constatant le coût élevé des logements, il notait que :

Les " ex " sont parfois contraints de demeurer ensemble, de s'endurer exacerbant ainsi le risque de violence; les femmes finissent par avoir peu de ressources pour s'en sortir. (Département des Services sociaux, 2013, p. 9-10)

Enfin, comment se mettre en sécurité, sans tout quitter, transport, emploi, logement... "Un des plus grands problèmes que nous rencontrons dans nos services, c'est justement qu'elles sont généralement des partenaires d'entreprise avec leur conjoint. C'est souvent une ferme héritée de plusieurs générations. [...] Puis aussi, elles savent que leur choix, si elles quittent, la ferme devra être vendue probablement, va être démantelée, et ça, c'est comme inacceptable. C'est un obstacle quasiment infranchissable. Alors que ce qu'elles auraient voulu choisir, c'est de quitter. Mais elles sentaient qu'elles ne pouvaient pas. C'est assez terrible. Attachées par ça, cet héritage familial et leur amour pour la ferme aussi. C'est le fait que c'est leur travail, c'est leur identité, c'est la fierté de leur travail, l'attachement et la pérennité de l'entreprise familiale aussi. »

Les intervenantes soulignent que pour les femmes, en matière économique, les défis sont presque insurmontables. Les défis de se réinstaller dans un milieu majoritairement agricole ou encore dans une ville où le taux de chômage est très élevé, 8,6 \% en 2011 à Hawkesbury, c'est quelquefois être condamnée à l'exil et au déracinement. Et après la séparation, la violence conjugale ne prend pas fin. Ainsi, plusieurs éléments font en sorte qu'elle se poursuit. Pensons entre autres aux conditions des visites ou à la garde des enfants. Souvent, le manque de ressources pour les visites supervisées fait en sorte que la belle-famille devient la ressource obligée. Quant aux conjointes, elles se retrouvent alors sous la surveillance de la belle-famille. Autre élément, en milieu rural, assurer sa sécurité est plus difficile. Les ex-conjoints connaissent la nouvelle adresse et sont souvent au courant des fréquentations de leur ex-conjointe. Et comment échapper au jugement et au stigmate social, comme le soulignent nombre d'intervenantes :

«En milieu rural, justement, tout le monde sait... Elle ne peut pas se cacher, tout le monde sait où elle est, qui elle est, tout le monde le lui rappelle, elle est super vulnérable : transport, enfants." 
"Les visites se font surtout dans le réseau de la famille, chez une des grandmères, ce qui peut augmenter le peu de soutien que les mères reçoivent et même les mettre en danger. Et ces conflits avec la belle-famille peuvent durer des années. "

"Des services pour les visites surveillées ne sont disponibles qu’à Hawkesbury, donc les personnes qui restent plus éloignées, leurs problèmes de transport augmentent aussi pour assurer leur propre sécurité. Si elles voulaient aller reconduire l'enfant plus loin, du fait du manque de transport, ça pourrait être plus difficile pour elles d'assurer leur sécurité. »

Parmi les conséquences de la violence conjugale, les problèmes de santé mentale sont souvent évoqués (Cory, et collab., 2010), mais les liens entre la violence conjugale, la santé mentale et les abus de substances en milieu rural sont moins connus. En 2008, dans les comtés de Grey et de Bruce, un comité s'est penché sur les besoins des femmes en milieu rural, vivant des situations de violence et ayant des problèmes de santé mentale et d'abus de substance. Cette recherche a fait ressortir entre autres que :

Les femmes ayant ou ayant eu des partenaires violents ont déclaré avoir été intimidées, avoir subi en quelque sorte un lavage de cerveau, les contrôlant au point où elles ne pouvaient reconnaître les situations de violence qu'elles vivaient, ni les liens entre ces situations et leurs problèmes de santé mentale/consommation. [notre traduction] (Rural Strategies for Women with Abuse, Mental Health, and Addiction Issues Project, 2008, p. 34)

Elles ont noté aussi que la stigmatisation et la honte accompagnaient les femmes qui vivaient des expériences de violence et qui étaient aux prises avec des problèmes de santé mentale, de toxicomanie. Ces situations rendaient difficile l'accès même aux services. Enfin, vivre dans une collectivité rurale renforçait le sentiment de honte.

Les intervenantes de Prescott-Russell font face à ces problématiques complexes des femmes et, connaissant la pénurie de services en santé mentale et en toxicomanie et les longues listes d'attente dans les comtés, elles sont d'avis qu'il n'en faut pas plus pour augmenter leur vulnérabilité. Cependant, la question de l'accès aux services en français n'est pas en soi une question centrale dans les comtés unis contrairement aux autres régions de l'Ontario puisque cette région est en fait une région majoritairement francophone; l'accès à une gamme de services en français s'est même élargi au cours des dernières années. Par ailleurs, la question se pose lorsque les femmes aux prises avec la 
violence conjugale doivent quitter leur milieu de vie pour aller à Ottawa. Là, la situation se corse et elle pourrait même être un frein à la décision de quitter le milieu. En effet, les ressources en français à Ottawa sont difficiles d'accès, ce qui oblige certaines femmes à recourir aux services en anglais. Dans une recherche récente, réalisée dans plusieurs régions ontariennes, nous avons pu faire ressortir certains des défis rencontrés, dont les délais d'attente pour les services de soutien pour elles et leurs enfants, le manque de places dans les maisons d'hébergement entre autres (Lapierre, et collab., 2014).

La violence conjugale touche les femmes de toutes les classes sociales et de tous les âges, mais elle est particulièrement présente chez les jeunes femmes « âgées entre 15 et 24 ans [...] avec le taux le plus élevé de violence entre partenaires amoureux " (Statistique Canada, 2013, p. 61). La Coalition s'intéressait plus particulièrement à la violence dans les fréquentations chez les jeunes filles. Les intervenantes et intervenants consultés lors d'un groupe d'entrevues ont noté la fréquence de la violence verbale, mais aussi sa banalisation et son invisibilisation sous le couvert de la jalousie, une preuve tangible d'amour selon les jeunes filles qui la subissent. Elle serait aussi plus insidieuse en milieu rural, car les milieux sont plus petits, les pressions qu'exercent les pairs sont plus présentes, tant pour empêcher la dénonciation que pour ne pas adopter de comportements différents de ceux socialement construits comme masculins. La violence sexuelle est aussi présente, mais, en milieu rural, elle prend des airs et un visage particuliers. Pour sortir, les jeunes filles, étant privées de transport en commun, doivent souvent recourir à des services d'amis... "Si elles veulent du transport, c'est [en échange] de petites faveurs ", comme le soulignent les intervenantes. Mais, c'est aussi l'espoir de pouvoir s'en sortir qui s'étiole et qui laisse les jeunes filles dans l'impuissance et le non-dévoilement. Un autre élément dont témoignaient les intervenantes auprès des jeunes filles, c'est la fréquence d'un passé d'abus sexuel dans l'enfance chez les jeunes filles vivant de la violence dans leurs fréquentations.

\section{Un portrait assez sombre des formes de violence vécues par les femmes dans les comtés}

La Coalition de Prescott-Russell pour éliminer la violence faite aux femmes a demandé aux organismes membres de remplir un questionnaire, le sondage éclair, pour chacune des clientes servies au cours de la période de collecte des données entre le 19 et le 23 novembre 2012. Ce questionnaire était rempli par une intervenante formée par la Coalition. Le calendrier de la collecte a été adapté aux réalités de chacun des organismes participants. Or, la durée de la collecte des données a été d'une semaine pour la Maison 
Interlude, mais de 24 heures pour l'Hôpital Général de Hawkesbury, ou encore de trois jours pour Valoris pour enfants et adultes de Prescott-Russell ou pour Ontario au travail. L'échantillon original était composé de 120 participantes. Parmi ces 120 participantes, $100(82,6 \%)$ ont déclaré avoir vécu une ou plusieurs formes de violence. Les données présentées sont propres aux 100 participantes qui se sont identifiées comme ayant vécu une ou plusieurs formes de violence.

Commençons par présenter quelques données sociodémographiques. Les participantes au sondage sont âgées de 16 à 74 ans. Parmi celles-ci, 81,8 \% ont le français comme langue maternelle (Coalition, 2014, p. 130). Les participantes ont répondu être nées au Canada dans une proportion de $97,9 \%$, et toutes les participantes, sauf une, ont répondu avoir la citoyenneté canadienne. Moins de $10 \%(8,1 \%)$ ont répondu appartenir à une minorité visible, et $3 \%$, être Autochtones (Coalition, 2014, p. 131). Enfin, en ce qui concerne la situation familiale, $40 \%$ d'entre elles vivent seules avec des enfants et $23 \%$ vivent en couple avec ou sans enfants (Coalition, 2014, p. 132). Parmi celles qui ont des enfants, la majorité en a deux (Coalition, 2014, p. 133). Si l'on compare cette population avec celle des comtés, il faut constater que les familles monoparentales ayant des femmes chefs de famille sont très minoritaires dans les comtés, ce qui peut être une source de marginalisation sociale dans un milieu assez homogène en matière de structure familiale. De la même manière, elles sont très majoritairement locataires, un renversement de la tendance générale observée dans les comtés, où les résidents occupent un logement qu'ils possèdent (Coalition, 2014, p. 133). Ces femmes sont aussi confrontées à des problèmes de très faible revenu, et la situation des mères monoparentales est assez critique puisque $28 \%$ d'entre elles ont un revenu de moins de $10000 \$$ par année et $38 \%$, un revenu entre 10000 \$ et 22000 \$ (Coalition, 2014, p. 135).

Nous nous sommes intéressées aux formes de violence qu'elles avaient vécues dans la dernière année et dans le passé. Fait à noter, $84 \%$ des femmes qui ont utilisé l'un ou l'autre des services de la région au moment du sondage ont vécu de la violence conjugale dans le passé : $43 \%$, de la violence dans leurs fréquentations lorsqu'elles avaient 25 ans et moins; $54 \%$, de la violence sexuelle, dont $42 \%$ dans leur enfance et $35 \%$ dans leur vie adulte en dehors du cadre conjugal, où ce pourcentage est de $46 \%$ (Coalition, 2014, p. 139). Il n'est pas possible de comparer ces données aux données publiées par Statistique Canada puisque les outils de recherche ne sont pas les mêmes. Mais force est de constater que les répondantes ont vécu plusieurs formes de violence, et ce, tout au cours de leur vie. En effet, en ce qui a trait uniquement aux formes de violence conjugale, la moitié des répondantes en ont vécu trois, soit la violence verbale, psychologique et physique, 
dans leur passé (Coalition, 2014, p. 142). Et c'est plus du quart (27\%) qui ont vécu, dans le passé, cinq formes de violence, la violence sexuelle et la violence économique ${ }^{1}$ s'ajoutant aux trois autres formes (Coalition, 2014, tableau 8.17, p. 142). On peut penser alors que la juxtaposition de ces formes de violence constituerait une forme d'escalade. Par ailleurs, dans le cadre de notre sondage, plusieurs des répondantes ont indiqué que ces violences sexuelles sont survenues à différents moments de leur vie, et qu'elles ont vécu des épisodes multiples de violence, de l'enfance à la vie adulte et dans leur relation de couple. Ces données sont assez dramatiques, et l'on peut estimer que ce n'est que la pointe de l'iceberg, sachant qu'en fait, la violence sexuelle ou encore les agressions sexuelles sont le plus souvent sous-déclarées (Publication de recherche de la Bibliothèque du Parlement, 2013).

Le dernier élément du sondage que nous avons abordé est centré sur le dernier événement de violence. Dans les situations de violence conjugale, la majorité des répondantes ont eu recours aux services policiers. Dans un peu plus de la moitié des cas signalés à la police, les agresseurs ont été arrêtés et, dans plus des deux tiers des cas, des accusations ont été portées (Coalition, 2014, tableau 8.20, p. 146). Notons aussi que, dans $34 \%$ des situations, les femmes avaient subi des blessures physiques et, dans la moitié des cas, des soins médicaux avaient été requis. Outre les blessures physiques, les femmes soulignaient qu'elles avaient, à la suite du dernier épisode, eu besoin d'un soutien psychologique, et ce, pour plus de 76 \% d'entre elles. Enfin, la moitié des femmes qui ont des enfants ont sollicité du soutien pour eux à la suite du dernier épisode de violence qu'elles avaient vécu (Coalition, 2014, p. 146).

Bien que les comparaisons avec les enquêtes de Statistique Canada présentent des défis importants, nous avons tout de même noté que deux éléments dans le recours aux services distinguent les réponses du sondage d'avec les résultats des données canadiennes, à savoir l'utilisation plus importante des services policiers et le recours aux membres de la famille ou aux amies et amis. En effet, le taux de signalement à la police est supérieur à celui fondé sur les données canadiennes, qui était de $30 \%$ en 2009, soit moins qu'en 2004, où il était de $36 \%$ (Statistique Canada, 2013, p. 10, cité dans Coalition, 2014, p. 146). Cependant, le signalement à la police est souvent fonction de la gravité des blessures. Autre élément à considérer : ce serait plus de $80 \%$ des femmes canadiennes victimes de violence conjugale pour qui « la famille et les amis ou les voisins étaient les sources les plus communes de soutien " (Statistique Canada, 2013, p. 93). Cependant, seulement $56 \%$ des répondantes au sondage ont eu recours aux services informels des amies et amis et de la famille. Quant aux services formels utilisés le plus fréquemment, 
les répondantes notent les services policiers, les maisons d'hébergement, les services de santé mentale et les services communautaires. Et dans leur recherche d'aide, elles utilisent plus d'un service pour les incidents de violence. Est-ce que l'on pourrait penser que le dévoilement ou la recherche de soutien informel auprès d'un membre de la famille sont plus difficiles en milieu rural ou même dans la région pour les femmes victimes de violence conjugale? Ajoutons que $3 \%$ des femmes n’ont fait appel à personne à la suite du dernier incident de violence. Est-ce parce qu’elles réussissent à résoudre la situation par elles-mêmes, ou parce qu'elles sont si honteuses qu'elles se taisent? On ne saurait conclure définitivement pour l'une ou l'autre de ces explications.

\section{Impacts de la recherche-action}

La recherche-action a eu des impacts intéressants tant sur les intervenantes et les organismes de service que sur la communauté des comtés. Tout d'abord, en ce qui concerne les intervenantes membres de la Coalition, notons que le rôle actif qu'elles ont eu dans le déroulement de la recherche a été un élément important dans la reconnaissance de leur expertise en matière de violence faite aux femmes et aux enfants tant au sein de leur organisme que dans la collectivité. Mais plus encore, elles tenaient à s'exprimer sur la dynamique de la violence en milieu rural pour les femmes particulièrement dans leur communauté. Ce partage était essentiel afin de briser leur isolement, mais aussi en vue de renforcer leur analyse féministe commune.

De plus, les nombreuses discussions relatives aux questions à inclure dans les groupes d'entrevues ou encore dans le sondage éclair leur ont permis de mettre en commun leurs questionnements sur les situations individuelles auxquelles elles font face et de raffiner leur analyse des enjeux auxquels elles sont confrontées. En effet, ce temps consacré à la recherche lors des rencontres trimestrielles de la Coalition leur permettait d'aller au-delà du partage sur les aspects plus quotidiens de leurs services respectifs et de se recentrer sur les espaces de collaboration et de partage des expertises. Et d'avoir fait le choix de devenir enquêtrice auprès de leur organisme, même le temps de 24 heures ou encore de 5 jours, leur a donné un espace de réflexion essentiel sur la dynamique des violences faites aux femmes. Ainsi, le questionnaire les sortait du cadre habituel de leur rencontre, loin des formulaires habituels ou encore des suivis à faire, ou encore il leur permettait d'ouvrir les rencontres spécifiquement sur les questions de violence, même si le but de la rencontre était tout autre pour la personne qui se présentait durant cette période à leur organisme. Cet espace de réflexion au sein de leur organisme a été aussi investi davantage 
lorsque nous avons fait appel à leur rétroaction sur leurs pratiques quotidiennes auprès des femmes vivant des situations de violence conjugale et sexuelle, en les invitant à partager les impacts et les limites de leurs interventions dans le processus judiciaire ou encore la garde des enfants. Enfin, le partage des recherches les plus récentes sur diverses thématiques, telles que l'abus de substance, le phénomène de la double accusation ${ }^{2}$, les modèles de prévention ou encore l'analyse des diversités des expériences des femmes, a été aussi très important pour s'éduquer collectivement en tant que membre de la Coalition.

Le processus de recherche au sein de chacun des organismes membres de la Coalition a renforcé les intervenantes dans leur volonté d'engager leur organisme dans la mission de la Coalition. Cet engagement s'est manifesté de plusieurs manières, que ce soit par le processus interne de demande d'autorisation de collaboration de leur organisme ou encore par les heures de formation exigée pour les participantes-enquêtrices, sans compter la disponibilité des intervenantes pour les dîners de recherche. Le caractère formel de la recherche permettait aussi de les ancrer dans une démarche plus structurée et "scientifique » sur leurs interventions et leurs préoccupations, en soi un processus de validation. Ce caractère formel renforçait aussi le bien-fondé de leurs préoccupations auprès de leur organisme. C'est comme si l'engagement de leur organisme validait leur engagement personnel auprès de la Coalition, et ce, réciproquement. En soi, le fait que tous les organismes membres aient partagé les informations requises, dans les délais requis et avec enthousiasme, leur a permis de réaliser que la Coalition est un organisme dynamique et jugé essentiel dans la communauté.

D'autres retombées de la recherche sont aussi étonnantes. Certains organismes de service de santé mentale ou de services sociaux qui ne sont pas membres de la Coalition, mais qui offrent des services aux femmes aux prises avec des situations de violence, ont participé à la recherche-action. Ces organismes ont accepté volontiers d'y prendre part, et cet exercice leur a en quelque sorte permis une prise de conscience de certaines réalités de violence touchant les personnes qui ont recours à leurs services. Ainsi, certains organismes se sont rendu compte que leur questionnaire d'accueil ou encore de suivi ne permettait pas le dépistage systématique de la violence faite aux femmes et aux enfants, et ce, malgré le fait qu'ils sont conscients que ces réalités les touchent directement. D’autres étaient même surpris d'apprendre que, malgré leur connaissance de plusieurs situations de violence conjugale ou sexuelle, des dossiers étaient peu documentés systématiquement et spécifiquement et, de ce fait, ne pouvaient être comptabilisés dans les données colligées par leur organisme. Enfin, d'autres organismes se sont rendu compte qu'ils ne colligeaient pas systématiquement ces expériences du fait que leur mandat était trop large, tout en 
reconnaissant que ces problématiques avaient des effets très négatifs pour la reprise de pouvoir tant recherchée des personnes auprès de qui ils interviennent. Par ailleurs, certains organismes ont aussi manifesté leur difficulté à prendre en compte ces situations en raison du manque de ressources spécifiques ou encore de leur interprétation des liens avec leur mandat. Par ailleurs, certains organismes ont réalisé jusqu'à quel point leurs outils de contextualisation des situations étaient différemment utilisés, selon les personnes qui étaient à l'accueil ou celles qui offraient directement des services. En effet, certaines situations passaient sous le radar. Ces différentes prises de conscience sont essentielles à la création d'un réel engagement de la communauté des comtés en vue de l'élimination de la violence faite aux femmes.

Et dernier point à souligner, durant la période de la recherche, la Coalition a poursuivi son travail de sensibilisation et a entrepris un travail très important auprès de la Corporation des Comtés unis. C'est ainsi que dans ce cadre, le 6 décembre 2013, le Conseil de la Corporation des Comtés unis de Prescott et Russell a adopté une résolution qui désigne le 6 décembre « la journée de la NON-VIOLENCE dans Prescott et Russell ». Cette recherche a ainsi permis d'ancrer davantage les membres dans leur communauté, d'assurer leur crédibilité en tant qu'organisme citoyen et de s'assurer de la légitimité de leurs actions.

\section{Notes}

1 «L'exploitation financière ou économique se produit lorsque : le partenaire défend à la femme de travailler; qu'il exerce un contrôle sur son choix de travail; qu'il l'empêche de devenir ou de rester autonome au plan financier; qu'il lui refuse tout accès à des ressources financières et l'exploite sur le plan financier. " (Les ministres responsables de la condition féminine à l'échelle fédérale, provinciale et territoriale, 2002, p. 3).

2 «Il y a accusation double lorsque la police porte accusation contre les deux partenaires par suite d'un incident de violence conjugale. [...] Lorsqu'une femme victime de violence est mise en accusation au même titre que son agresseur, l'accusation double est une conséquence imprévue et indésirable de la politique de mise en accusation obligatoire. Afin d'éviter la mise en accusation des victimes qui agissent par légitime défense, les services de police partout en Ontario examinent le concept «d'agresseur dominant» dans les incidents de violence conjugale. " (Centre for Children and Families in the Justice System, 2005, p. 11) 


\section{Bibliographie}

BROWNRIDGE, Douglas A. (2009). Violence Against Women: Vulnerable Populations, New York, Routledge, $296 \mathrm{p}$.

CEVAW (2012). Niagara Region Domestic Violence Report Card 2011/12, réf. du 15 janvier 2016, http://cevaw.com/niagara-region-domestic-violence-report-card-201112/ http://www.supportniagara.ca/

CENTRE FOR CHILDREN AND FAMILIES IN THE JUSTICE SYSTEM (2005). Enseigner en quoi consiste la violence faite aux femmes et ses effets sur les enfants, par Linda Baker et Alison Cunningham, London, réf. du 13 juillet 2016, www.lfcc.on.ca/guide_professeur.html

COALITION D'OTTAWA CONTRE LA VIOLENCE FAITE AUX FEMMES (2011). À l'abri des regards, volume II : Prévalence de la violence faite aux femmes à Ottawa, Ottawa, 54 p., réf. du 15 janvier 2016,

http://www.octevaw-cocvff.ca/sites/all/files/pdf/reports/HiddenFromSight-FR-2013.pdf

COALITION D'OTTAWA CONTRE LA VIOLENCE FAITE AUX FEMMES (2009). À l'abri des regards, volume I : Prévalence de la violence faite aux fermmes à Ottawa, Ottawa, 34 p., réf. du 15 janvier 2016,

http://www.octevaw-cocvff.ca/sites/all/files/pdf/fr/reports/Hidden_from_Sight.pdf

COALITION DE PRESCOTT-RUSSELL POUR ÉLIMINER LA VIOLENCE FAITE AUX FEMMES (2014). Une réalité qu'on ne peut ignorer: Portrait régional des violences faites aux femmes des comtés de Prescott-Russell et les services communautaires disponibles pour les femmes et les familles, Hawkesbury, 174 p., réf. du 15 janvier 2016,

http://coalitionviolencepr.ca/images/client/documents/Documents_pertinents/Rapport_final__14mai_2014.pdf

CONSEIL DES COMTÉS UNIS DE PRESCOTT-RUSSELL (2013). Résolution du conseil, Le 6 décembre désigné la journée de la non-violence dans Prescott et Russell, Bulletin des comtés, réf. du 26 février 2016,

http://www.prescott-russell.on.ca/fr/component/content/article/42-global-site-news/portal-homepage-news-francais/963-le-6-decembre-designe-la-journee-de-la-non-violence-dans-prescott-etrussell

CORY, Jill, et collab. (2010). Building Bridges: Linking Woman Abuse, Substance Use and Mental Ill Health Summary Report, réf. du 15 janvier 2016,

http://www.canadianwomen.org/sites/canadianwomen.org/files/PDF\%20-\%20VP\%20 Resources\%20-\%20BCSTH\%20CWF\%20Report_Final_2011_\%20Mental\%20Health_ Substance\%20use.pdf 
DEKESEREDY, Walter, et Martin SCHWARTZ (2009). Dangerous Exits: Escaping Abusive Relationships in Rural America, New Brunswick, New Jersey, Rutgers University Press, 272 p.

DEKESEREDY, Walter, Molly DRAGIEWICZ et Callie Marie RENNISON (2012). «Racial/Ethnic Variations in Violence Against Women: Urban, Suburban, and Rural Differences ", International Journal of Rural Criminology, Vol. 1, № 2, p. 184-202.

DÉPARTEMENT DES SERVICES SOCIAUX, Comtés unis de Prescott-Russell (2013). Un logement pour tous. Le logement abordable et la lutte à l'itinérance. Rapport préliminaire, Hawkesbury, Document miméo.

DOHERTY, Deborah (2002). Mettre l'information sur le droit en matière de violence familiale à la disposition des gens vivant en milieu rural. Un répertoire de pratiques prometteuses, Centre Muriel McQueen Fergusson pour la recherche sur la violence familiale, ministère de la Justice du Canada, réf. du 15 janvier 2016, http://canada.justice.gc.ca/fr/ps/fm/reports/fv_rural_f.pdf

DOHERTY, Deborah, et Jennie HORNOSTY (2008). « La culture des armes à feu en milieu rural : impact sur la violence contre les femmes ", Violences faites aux fermmes, Collection Problèmes sociaux et interventions sociales, Québec, PUQ, p. 17-37.

DRAGIEWICZ, Molly, et Walter DEKESEREDY (2008). "Étude sur les expériences des femmes victimes de violence ayant recours au tribunal de la famille dans huit régions de l'Ontario ", Toronto, Imprimeur de la Reine, réf. du 15 janvier 2016,

http://www.citizenship.gov.on.ca/owd/french/resources/publications/dvac/etude.html

HORNOSTY, Jennie, et Deborah DOHERTY (2002). Responding to Wife Abuse in Farm and Rural Communities: Searching for Solutions that Work, SIPP Public Policy Paper No. 10, présenté en 2001 dans le cadre de la conférence Rural Canada: Moving Forward or Left Behind?, Regina, Saskatchewan Institute of Public Policy.

JIWANI, Yasmin, Patricia KACHUK et Shelley MOORE (1998). "Rural Women and Violence: A Study of Two Communities in British Columbia », Justice Canada, réf. du 15 janvier 2016,

http://fredacentre.com/wp-content/uploads/2010/09/Jiwani-1998-combined.pdf

KASDORFF, Deborah, et Barbara ERB (2010). Serving Victims of Violence in Rural Communities. Challenges and Best Practices, préparé pour le Victim/Witness Assistance Program, East Region, 20 p.

http://library.queensu.ca/files/Rural\%20Issues\%20in\%20Eastern\%20Ontario\%20Final\%20 Jan\%2010\%203.pdf

LAPIERRE, Simon, et collab. (2014). «Quand le manque d'accès aux services en français revictimise les femmes victimes de violence conjugale et leurs enfants ", Reflets : revue d'intervention sociale et communautaire, Vol. 20, No 2, p. 22-51. 
LES MINISTRES RESPONSABLES DE LA CONDITION FÉMININE À L'ÉCHELLE FÉDÉRALE, PROVINCIALE ET TERRITORIALE (2002). Évaluation de la violence contre les femmes : un profil statistique, Ottawa, http://www.gov.pe.ca/photos/original/iws_stat_prof_f.pdf

MARTZ, Diane J. FORSDICK, et Deborah BRYSON SARAUER (2001). « Politiques ascendantes : les services ruraux pour les femmes ayant survécu à la violence ", Bulletin de recherche des centres d'excellence pour la santé des fermmes, Vol. 2, № 1, p. 14-16, réf. du 15 janvier 2016, http://www.cwhn.ca/sites/default/files/CEWH-bulletin/bulletin/fv2n1/page8.html

MARTZ, Diane J. FORSDICK, et Deborah BRYSON SARAUER (2000). Domestic Violence and the Experiences of Rural Women in East Central Saskatchewan, réf. du 15 janvier 2016, http://www.pwhce.ca/pdf/domestic-viol.pdf

MOSHER, Janet, en collaboration avec Patricia EVANS et Margaret LITTLE (2004). Une marche en terrain glissant: Les femmes maltraitées et leurs expériences avec le système du bien-être social de l'Ontario. Rapport final sur les résultats de recherche du Projet de recherche sur la femme et la violence à l'intérieur du bien-être social, Partenaires communautaires : Eileen Morrow (Ontario Association of Interval and Transition Houses); Jo-Anne Boulding et Nancy VanderPlaats (Ontario Social Safety Network), réf. du 15 janvier 2016,

https://apps.osgoode.yorku.ca/osgmedia.nsf/0/C4A73A038D3150848525709A005403F0/\$FI LE/Walking\%20on\%20Eggshells\%20french.pdf

ONTARIO RURAL WOMEN ABUSE STUDY (2000). Étude sur la violence envers les femmes en milieu rural ontarien, [rapport de recherche], par Lorri Biesenthal, Lynne Dee Sproule, Mary Nelder, Susan Golton, Donna Mann, Denise Podovinnikoff, Inge Roosendaal, Shellie Warman et Donna Lunn. En collaboration avec Community Abuse Programs of Rural Ontario, réf. du 15 janvier 2016, http://www.justice.gc.ca/fra/pr-rp/jp-cj/victim/rr00_15/rr00_15.pdf

PEEK-ASA, Corinne, et collab. (2011). "Rural Disparity in Domestic Violence Prevalence and Access to Resources ", Journal of Women's Health, Vol. 20, No 11, p. 1743-1749.

PUBLICATION DE RECHERCHE DE LA BIBLIOTHÈQUE DU PARLEMENT (2013). Agressions sexuelles : sous-signalement et faible taux de condamnation, par Julia Nicol, Division des affaires juridiques et sociales, le 17 avril 2013, Note de la Colline n ${ }^{\circ}$ 2013-16-F, réf. du 15 janvier 2016, http://www.parl.gc.ca/Content/LOP/ResearchPublications/2013-16-f.htm

RIDDELL, Thelma, Marilyn FORD-GILBOE et Beverly LEIPERT (2008). "Strategies Used by Rural Women to Stop, Avoid, or Escape From Intimate Partner Violence », Health Care for Women International, Vol. 30, № 1-2, p. 134-159.

RURAL STRATEGIES FOR WOMEN WITH ABUSE, MENTAL HEALTH, AND ADDICTION ISSUES PROJECT (2008). No Wrong Door: Creating a Collaborative Rural Response for Women 
with Abuse, Mental Health and Addictions Issues, réf. du 15 janvier 2016, http://www.endabusenow.ca/files/Final\%20Report\%20No\%20Wrong\%20Door\%20.pdf

SCOTT, Wendy, et Carolyn VANDINE (1995). Family Violence in Rural, Farm and Remote Canada, The Canadian Farm Women's Network, ministère de la Justice du Canada, réf. du 15 janvier 2016, http://www.acjnet.org/docs/famvidoj.html

STATISTIQUE CANADA (2013). "Mesure de la violence faite aux femmes : tendances statistiques ", dans Maire Sinha (dir.), Juristat, 130 p., réf. du 15 janvier 2016, http://www.statcan.gc.ca/pub/85-002-x/2013001/article/11766/11766-4-fra.htm\#a1

VILLE DE HAWKESBURY (2015). Tableau : Taux d'emploi 2011, réf. du 15 janvier 2016, http://www.hawkesbury.ca/index.php/fr/developpement-economique/demographie 